\title{
Synthesis of some novel fluorinated 4-thiazolidinones containing amide linkages and their antimicrobial screening
}

\author{
Tejaskumar J. Shah and Vikas A. Desai* \\ Department of Chemistry, B. K. M. Science College, Valsad-396001 \\ (Affiliated to Veer Narmad South Gujarat University, Surat-395007, State-Gujarat, India) \\ E-mail: tejas_shah1977@yahoo.com
}

\begin{abstract}
Efficient syntheses of some 4-thiazolidinones are described. A series of 2-(substituted phenyl)-3[4-(2,4-dichloro-5-fluorophenyl)-6-(2-thienyl)pyrimidine-2-yl-ureido]-5H/methyl/carboxymethyl-4-thiazolidinones 5a-e,6a-e,7a-e were prepared. The structures of the synthesised compounds were assigned on the basis of elemental analysis, IR and ${ }^{1} \mathrm{H}$ NMR spectral data. All the products were screened against different strains of bacteria and fungi.
\end{abstract}

Keywords: Schiff base, 4-thiazolidinones, antibacterial activity, synthesis

\section{Introduction}

The presence of a pyrimidine nucleus in compounds, often leads to exceptionally interesting biological and pharmacological activities. 4-Thiazolidinones have been reported to show a broad spectrum of biological activities. ${ }^{1-10}$ The pharmacological properties of 4-thiazolidinones encouraged our interest in synthesizing several new compounds featuring various heterocyclic rings, attached to 4-thiazolidinone moieties. As a part of our aim to search for biologically active heterocycles containing sulfur and nitrogen, we have now synthesised a series of 2-(substituted phenyl)-3-[4-(2,4-dichloro-5-fluorophenyl)-6-(2-thienyl) pyrimidine-2-yl-ureido]-5H / methyl / carboxymethyl- 4-thiazolidinones 5a-e, 6a-e, 7a-e.

2,4-Dichloro-5-fluoroacetophenone on reaction with thiophene-2-carbaldehyde in a ClaisenSchmidt condensation ${ }^{11-13}$ gave 1-(2,4-dichloro-5-fluorophenyl)-3-(2-thienyl)-2-propen-1-one 1 (Scheme 1), which on reaction with guanidine nitrate and $40 \% \mathrm{KOH}$ yielded 2-amino-4-(2,4dichloro-5-fluorophenyl)-6-(2-thienyl)pyrimidine 2 (Scheme 2) in 70\% yield. 
<smiles>CC(=O)c1cc(F)c(Cl)cc1Cl</smiles><smiles>O=Cc1cccs1</smiles><smiles>O=C(/C=C/c1cccs1)c1cc(F)c(Cl)cc1Cl</smiles>

Scheme 1<smiles>N=C(N)NNC(=O)OC(=O)NN</smiles>

\section{Scheme 2}

Compound 2 on further reaction with methyl chloroformate (MCF) followed by reaction with hydrazine hydrate gave 4-[4-(2,4-dichloro-5-fluorophenyl)-6-(2-thienyl)pyrimidin-2-yl] semicarbazide 3 in $68 \%$ yield. Compound 3 on condensation with various substituted aromatic aldehydes afforded 1-(substituted benzylidene)-4-[4-(2,4-dichloro-5-fluorophenyl)-6-(2thienyl)pyrimidin-2-yl] semicarbazides 4a-e (Scheme 3). These imines on cyclocondensation with mercaptoacetic acid $(\mathrm{X}=\mathrm{H}), 2$-mercaptopropanoic acid $\left(\mathrm{X}=\mathrm{CH}_{3}\right)$ or mercaptosuccinic acid $\left(\mathrm{X}=\mathrm{CH}_{2} \mathrm{COOH}\right)$ respectively led to the formation of 2-(aryl)-3-[4-(2,4-dichloro-5fluorophenyl)-6-(2-thienyl)pyrimidin-2-yl-ureido]-5H / methyl / carboxymethyl-4thiazolidinones 5a-e, 6a-e,7a-e (Scheme 3).

\section{Results and Discussion}

The results given in Table 1 confirm that compounds with 4-thiazolidinone units are potential antibacterial agents. The structures of the synthesised compounds were confirmed by elemental analysis, IR spectra and ${ }^{1} \mathrm{H}$ NMR spectral analysis. The IR spectra of 5a-e, 6a-e, 7a-e exhibited a band due to $=\mathrm{CH}$ str. $\left(3100-3000 \mathrm{~cm}^{-1}\right), \mathrm{C}=\mathrm{C}$ str. $\left(1635-1495 \mathrm{~cm}^{-1}\right), \mathrm{C}-\mathrm{H}$ bending $[1,2,4,5-$ substituted (900-860 $\left.\mathrm{cm}^{-1}\right)$ ], $\mathrm{C}-\mathrm{H}$ bending [1,4-substituted aryl $\left.\left(840-800 \mathrm{~cm}^{-1}\right)\right], \mathrm{C}-\mathrm{Cl}$ str. (750$\left.700 \mathrm{~cm}^{-1}\right)$, C-F str. $\left(1100-1000 \mathrm{~cm}^{-1}\right), \quad \mathrm{C}-\mathrm{S}-\mathrm{C}$ str. $\left(700-600 \mathrm{~cm}^{-1}\right), \mathrm{C}=\mathrm{N}$ (ring) $\left(1650-1580 \mathrm{~cm}^{-1}\right)$ stretching vibration band, $\mathrm{C}=\mathrm{O}\left(1674 \mathrm{~cm}^{-1}\right.$, 4-thiazolidinone moiety $)$ which indicated the presence of a pyrimidine ring and a 4-thiazolidinone ring.

The IR spectrum of 1 showed a band at $1654 \mathrm{~cm}^{-1}$ due to the $\mathrm{C}=\mathrm{O}$, and a band at $1642 \mathrm{~cm}^{-1}$ due to the $\mathrm{C}=\mathrm{N}$ str. of the pyrimidine moiety and $3418 \mathrm{~cm}^{-1}$ due to $\mathrm{NH}_{2}$ in compound 2 . The 
appearance of band at $1610 \mathrm{~cm}^{-1}$ due to the -NH-CO-NH- unit in compound 3 proved the conversion of compound 2 to 3 . Similarly, the disappearance of a band at $3418 \mathrm{~cm}^{-1}\left(\mathrm{NH}_{2}\right)$ and the appearance of a band at $1600 \mathrm{~cm}^{-1}$ due to $\mathrm{C}=\mathrm{N}$ str. proved the conversion to compounds 4 a-e. The final structures 5a-e, 6a-e, 7a-e were established by the disappearance of the $\mathrm{C}=\mathrm{N}$ str. And the appearance of a band at $1674 \mathrm{~cm}^{-1}$ due to $\mathrm{C}=\mathrm{O}$ str. (4-thiazolidinone).

2

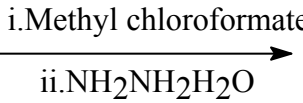<smiles>NNC(=O)Nc1nc(-c2cccs2)cc(-c2cc(F)c(Cl)cc2Cl)n1</smiles>

3

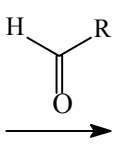<smiles>[R]C=NNC(=O)Nc1nc(-c2cccs2)cc(-c2cc(F)c(Cl)cc2Cl)n1</smiles>

4a-e

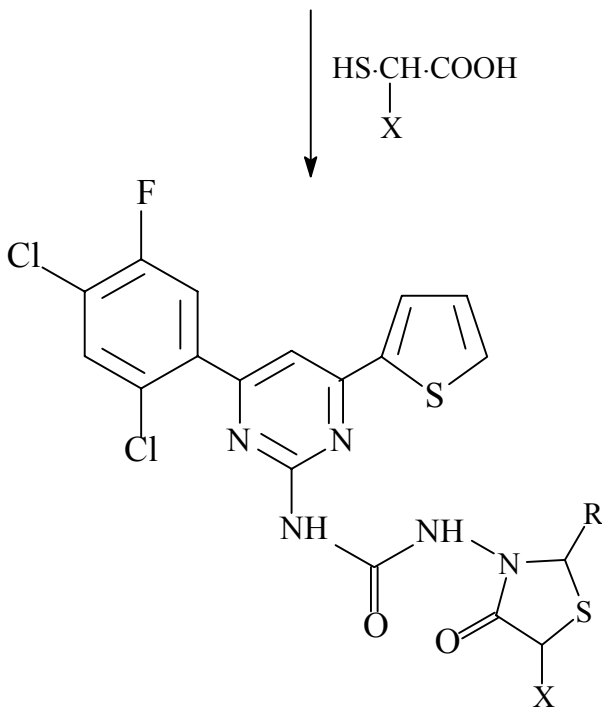

5a-e, 6a-e, 7a-e

Scheme 3. $\mathrm{R}=$ aryl; $\mathrm{X}=\mathrm{H}\left(\right.$ 5a-e), $\mathrm{CH}_{3}$ (6a-e), $\mathrm{CH}_{2} \mathrm{COOH}$ (7a-e).

In the ${ }^{1} \mathrm{H}$ NMR spectrum of 1 , the signal at $\delta 7.1$ corresponded to the $\mathrm{COCH}=\mathrm{CH}$ unit. A signal at $\delta 6.8$ for the $\mathrm{CH}$ (pyrimidine ring) verified the structure 2 . In the same way, the disappearance of the signal at $\delta 5.1$ due to $\mathrm{NH}_{2}$ and the appearance of a signal at $\delta 4.4$ due to $\mathrm{N}=\mathrm{CH}$ provided further evidence for the conversion of compound $\mathbf{3}$ into compound $\mathbf{4}$. Finally the 
disappearance of the $\mathrm{N}=\mathrm{CH}$ signal and the appearance of a signal at $\delta 6.1$ for the thiazolidinone ring $\mathrm{CH}$ showed the formation of compounds $5 \mathbf{a}-\mathbf{e}, \mathbf{6 a - e , 7 a - e . ~}$

Table 1. Antibacterial and antifungal activity of 5a-e, 6a-e, 7a-e

\begin{tabular}{|c|c|c|c|c|c|c|}
\hline \multirow[t]{4}{*}{ No. } & \multicolumn{3}{|c|}{ Antibacterial activity } & \multicolumn{2}{|c|}{ Antifungal activity } & \\
\hline & \multicolumn{4}{|c|}{ Diameter of Zone of Inhibition (in mm) } & \multicolumn{2}{|c|}{ Diameter of the MIC in $\mathrm{mm}$} \\
\hline & $\underline{\text { S. aureus }}$ & B. substilis & E. coli & P. aeruginosa & C. crusei & C. albicans \\
\hline & $\begin{array}{c}\text { ATCC } \\
6538\end{array}$ & $\begin{array}{l}A T C C \\
6633\end{array}$ & $\begin{array}{l}\text { ATCC } \\
8739\end{array}$ & $\begin{array}{l}\text { ATCC } \\
1539\end{array}$ & $\begin{array}{l}A T C C \\
14243\end{array}$ & $\begin{array}{l}\text { ATCC } \\
64550\end{array}$ \\
\hline $\mathbf{5 a}$ & ++ & ++ & ++ & + & ++ & ++ \\
\hline $5 \mathbf{b}$ & ++ & - & ++ & - & + & ++ \\
\hline $5 c$ & ++ & + & ++ & + & + & - \\
\hline $5 d$ & ++ & ++ & ++ & + & ++ & + \\
\hline $5 e$ & + & + & + & - & + & ++ \\
\hline $\mathbf{6 a}$ & ++ & ++ & + & + & - & + \\
\hline $\mathbf{6 b}$ & + & + & + & - & - & + \\
\hline $6 c$ & + & + & ++ & + & ++ & + \\
\hline 6d & ++ & ++ & + & + & ++ & + \\
\hline $6 e$ & ++ & ++ & ++ & + & + & ++ \\
\hline $7 \mathbf{a}$ & + & ++ & + & - & - & + \\
\hline $7 b$ & ++ & + & ++ & + & ++ & + \\
\hline $7 c$ & + & ++ & + & + & ++ & + \\
\hline $7 d$ & + & + & + & - & - & - \\
\hline $7 e$ & + & + & + & + & - & ++ \\
\hline Streptomycin & ++++ & ++++ & ++++ & ++++ & - & - \\
\hline Griseofulvin & - & - & - & - & ++++ & ++++ \\
\hline \multicolumn{7}{|c|}{ Diameter of zone of inhibition : $(-)<6 \mathrm{~mm},(+) 6-12 \mathrm{~mm},(++) 12-20 \mathrm{~mm},(+++) 20-25 \mathrm{~mm},(++++)$} \\
\hline Diameter of $\mathrm{t}$ & he MIC: (-) & $\mathrm{n},(+) 5-12$ & $\mathrm{~m},(++) 12$ & $8 \mathrm{~mm},(+++)$ & $-24 \mathrm{~mm},(+$ & +) $24-32 \mathrm{~mm}$ \\
\hline
\end{tabular}

\section{Antibacterial activity}

All the synthesised compounds were screened for their in vitro antibacterial activity against Escherichia coli (ATCC 8739), Pseudomonas aureginosa (ATCC 1539) and Staphylococcus aureus (ATCC 6538), Bacillus substilis (ATCC 6633) bacteria using the cup-plate agar diffusion 
method. ${ }^{14}$ Streptomycin was used as reference drug. The degree of inhibition varied with the test compound as well as with the bacterium.

Against Bacillus substilis (ATCC 6633), compounds 5a, 5d, 6d, 6e, 7a, 7c exhibited good antibacterial activity, whereas compounds $5 \mathbf{b}, \mathbf{5 c}, \mathbf{6 e}, \mathbf{6 c}, \mathbf{7 b}, 7 \mathbf{e}$ showed low activity. On the other hand Pseudomonas aureginosa (ATCC 1539) showed poor responses to five of the prepared products. Compounds 5d, 6d, 7c and compounds 5a, 6d, 7b exhibited good antibacterial activity towards Escherichia coli (ATCC 8739) and Staphylococcus aureus (ATCC 6538) respectively.

On the basis of the antibacterial activity data it could be concluded that some of the compounds possess considerable antibacterial activity due to the presence of methoxy, fluoro and chloro groups. However the activity of the tested compounds is less than that of streptomycin.

\section{Antifungal activity}

The compounds were tested for their antifungal activity using Candia crusei (ATCC 14243) and Candida albicans (ATCC 64550). For all compounds, the minimum inhibitory concentration (MIC) was taken as the lowest concentration at which there was $100 \%$ inhibition of growth compared with the growth for a drug-free control. ${ }^{15}$ Griseofulvin was used as reference drug for comparison. The data of antifungal activity (Table 1) showed that generally, the compounds 5a, 5d, 6c, 6d, 7b, 7c were more effective against Candia crusei (ATCC 14243), while compounds $\mathbf{5 b}, \mathbf{6 a}, \mathbf{6 b}, \mathbf{7 d}, \mathbf{7 e}$ exhibited low antifungal activity. Moreover, compounds 5a, 5b, 6e, 7e demonstrated good activity against Candida albicans (ATCC 64550). On the basis of the antifungal activity data it could be concluded that some of the compounds possess good activity. however, none of compounds was superior to standard used against any of the fungi.

\section{Experimental Section}

General Procedures. Melting points were determined in an open capillary tube and are uncorrected. Infrared (IR) Spectra were measured on a FTIR-8400 Shimadzu spectrometer using $\mathrm{KBr}$ pellets. The proton nuclear magnetic resonance $\left({ }^{1} \mathrm{H} \mathrm{NMR}\right)$ spectra were recorded on a Brüker Avance dpx-200 (at $200 \mathrm{MHz}$ ) spectrometer with $\mathrm{CDCl}_{3}$ as a solvent with tetramethylsilane (TMS) as an internal reference. Chemical shifts are expressed in $\delta$ ppm. Elemental analysis of $\mathrm{C}$ and $\mathrm{H}$ was performed by Central Drug Research Institute, Lucknow and nitrogen was estimated by Kjeldhal's method, ${ }^{16}$ results are within $\pm 0.4 \%$ of the theoretical value. Thin layer chromatography was carried out on silica gel (Kieselgel G; Merck) to monitor the reactions and to check the purity of the compounds. All reagents were of the highest purity available commercially. 
1-(2,4-Dichloro-5-fluorophenyl-3-(2-thienyl)-2-propen-1-one $\quad 1$ 2,4-dichloro-5-fluoroacetophenone $(0.01 \mathrm{~mol})$ and thiophene-2-carbaldehyde $(0.01 \mathrm{~mol})$ were stirred in methanol $(50.0 \mathrm{~mL})$ at $32{ }^{\circ} \mathrm{C}$ for $1 / 2 \mathrm{~h}$. Then, $10 \% \mathrm{KOH}$ solution $(3.0 \mathrm{~mL})$ was added to the reaction mass and it was stirred for a further $4 \mathrm{~h}$. After standing at room temperature for $24 \mathrm{~h}$, the reaction mixture was added to ice-cold water and acidified with dilute $\mathrm{HCl}$. The precipitate formed was filtered, washed with water, dried and recrystallized from ethanol to afford pure pale yellow product 1 in $81 \%$ yield. m.p. $92{ }^{\circ} \mathrm{C}$; IR $(\mathrm{KBr}):=\mathrm{CH}$ str. $\left(3080 \mathrm{~cm}^{-1}\right), \mathrm{C}=\mathrm{C}$ str. $\left(1517 \mathrm{~cm}^{-1}\right), \mathrm{C}-\mathrm{H}$ bending [1,2,4,5-substituted $\left.\left(889 \mathrm{~cm}^{-1}\right)\right], \mathrm{C}-\mathrm{H}$ bending [1,4-substituted $\left.\left(848 \mathrm{~cm}^{-1}\right)\right], \mathrm{C}-\mathrm{Cl}$ str. $(772$ $\left.\mathrm{cm}^{-1}\right), \mathrm{C}-\mathrm{F}$ str. $\left(1025 \mathrm{~cm}^{-1}\right), \mathrm{C}=\mathrm{O}\left(1654 \mathrm{~cm}^{-1}\right) ;{ }^{1} \mathrm{H}$ NMR $\left(\delta, \mathrm{CDCl}_{3}\right): 7.1(1 \mathrm{H}, \mathrm{dd}, \mathrm{COCH}=\mathrm{CH}), 7.3$ to 7.8 (5H, m, Ar-H); Anal. Calcd. for $\mathrm{C}_{13} \mathrm{H}_{7} \mathrm{Cl}_{2} \mathrm{OFS}$ : C, 51.83; H, 2.33\%. Found: C: 51.87; H: $2.39 \%$.

2-Amino-4-(2,4-dichloro-5-fluorophenyl)-6-(2-thienyl) pyrimidine 2. A mixture of 1-(2,4dichloro-5-fluorophenyl)-3-(2-thienyl)-2-propen-1-one $(0.01 \mathrm{~mol})$, guanidine nitrate $(0.01 \mathrm{~mol})$ and $40 \% \mathrm{KOH}(2.0 \mathrm{~mL})$ was refluxed in ethanol $(50.0 \mathrm{~mL})$ for $20 \mathrm{~h}$. After completion of reaction, the reaction mixture was cooled to room temperature, poured into crushed ice and water and acidified with dilute $\mathrm{HCl}$. The separated solid was filtered, washed with water, dried and recrystallized from ethanol to give the title compound as a white solid in $70 \%$ yield. m.p. $167{ }^{\circ} \mathrm{C}$; IR (KBr): $-\mathrm{NH}_{2}\left(3418 \mathrm{~cm}^{-1}\right), \mathrm{C}=\mathrm{N}$ str. (pyrimidine moiety, $\left.1642 \mathrm{~cm}^{-1}\right), \mathrm{C}=\mathrm{C}$ str. $\left(1572 \mathrm{~cm}^{-1}\right), \mathrm{C}-\mathrm{F}$ str. $\left(1056 \mathrm{~cm}^{-1}\right), \mathrm{C}-\mathrm{Cl}$ str. $\left(755 \mathrm{~cm}^{-1}\right) ;{ }^{1} \mathrm{H} \mathrm{NMR}\left(\delta, \mathrm{CDCl}_{3}\right): 5.15\left(2 \mathrm{H}, \mathrm{s}, \mathrm{NH}_{2}\right), 6.7(1 \mathrm{H}, \mathrm{s}$, pyrimidine $\mathrm{CH}$ ), 7.3-7.9 (m, 5H, Ar-H); Anal. Calcd. For $\mathrm{C}_{14} \mathrm{H}_{8} \mathrm{Cl}_{2} \mathrm{FN}_{3} \mathrm{~S}$ : C, 49.41; $\mathrm{H}, 2.35$; N, $12.35 \%$. Found: C, 49.46; H, 2.39; N, $12.30 \%$.

4-[4-(2,4-Dichloro-5-fluorophenyl)-6-(2-thienyl)pyrimidine-2-yl] semicarbazide 3. A mixture of 2-amino-4-(2,4-dichloro-5-fluorophenyl)-6-(2-thienyl)pyrimidine $(0.01 \mathrm{~mol})$ and methyl chloroformate $(0.02 \mathrm{~mol})$ was refluxed in the presence of triethylamine for $10 \mathrm{~h}$ in ethanol $(50.0$ $\mathrm{mL}$ ). After completion of reaction the reaction mixture poured into ice-cold water. The separated solid was acidified with dilute $\mathrm{HCl}$, filtered, washed with water and dried. The dried crude was refluxed with hydrazine hydrate in toluene for $7 \mathrm{~h}$. After reaction completion, the toluene was distilled under vacuum to dryness. The material was washed with water till neutral, dried and recrystallized from acetone as a white solid in $68 \%$ yield. m.p. $188{ }^{\circ} \mathrm{C}$; IR $(\mathrm{KBr}): \mathrm{NH}_{2}\left(3418 \mathrm{~cm}^{-}\right.$ $\left.{ }^{1}\right), \mathrm{C}=\mathrm{O}$ str. $\left(1660 \mathrm{~cm}^{-1}\right), \mathrm{C}=\mathrm{N}$ str. (pyrimidine moiety, $\left.1642 \mathrm{~cm}^{-1}\right), \mathrm{NH}-\mathrm{CO}-\mathrm{NH}\left(1610 \mathrm{~cm}^{-1}\right.$, urea), C-Cl str. $\left(755 \mathrm{~cm}^{-1}\right), \mathrm{C}-\mathrm{F}$ str. $\left(1056 \mathrm{~cm}^{-1}\right), \mathrm{C}-\mathrm{S}-\mathrm{C}$ str. $\left(740 \mathrm{~cm}^{-1}\right) ;{ }^{1} \mathrm{H}$ NMR $\left(\delta, \mathrm{CDCl}_{3}\right): 5.1(2 \mathrm{H}, \mathrm{s}$, NH-N $\left.\underline{H}_{2}\right), 6.8(1 \mathrm{H}, \mathrm{s}$, pyrimidine $\mathrm{CH}), 7.3-7.8(\mathrm{~m}, 5 \mathrm{H}, \mathrm{Ar}-\mathrm{H}), 8.4(1 \mathrm{H}, \mathrm{s}, \mathrm{CO}-\mathrm{NH})$; Anal. Calcd. for $\mathrm{C}_{15} \mathrm{H}_{10} \mathrm{~N}_{5} \mathrm{OCl}_{2} \mathrm{FS}$ : C, 45.23; H, 2.51; N, 17.59\%. Found: C, 45.18; H, 2.56; N, 17.52\%.

\section{1-(4-Methoxybenzylidene)-4-[4-(2,4-dichloro-5-fluorophenyl)-6-(2-thienyl)pyrimidine-2-yl]}

semicarbazide 4c. A mixture of 4-[4-(2,4-dichloro-5-fluorophenyl)-6-(2-thienyl)pyrimidin-2-yl] semicarbazide $3(0.01 \mathrm{~mol})$ and 4-methoxybenzaldehyde $(0.01 \mathrm{~mol})$ was refluxed in methanol $(60.0 \mathrm{~mL})$ in the presence of a catalytic amount of glacial acetic acid for $10 \mathrm{~h}$. After reaction completion, the reaction mass was cooled to room temperature, and poured onto ice-cold water with vigorous string. The separated solid was filtered, washed with 5\% sodium bisulfite solution to remove excess aldehyde and recrystallized from chloroform as a light yellow solid in $65 \%$ 
yield. m.p. $195{ }^{\circ} \mathrm{C}$; IR (KBr): $\mathrm{NH}\left(3270 \mathrm{~cm}^{-1}\right),=\mathrm{CH}$ str. $\left(3068 \mathrm{~cm}^{-1}\right), \mathrm{C}=\mathrm{O}$ str. $\left(1660 \mathrm{~cm}^{-1}\right), \mathrm{C}=\mathrm{N}$ str. (Schiff base, $\left.1600 \mathrm{~cm}^{-1}\right), \mathrm{C}-\mathrm{F}$ str. $\left(1056 \mathrm{~cm}^{-1}\right), \mathrm{C}-\mathrm{Cl}$ str. $\left(754 \mathrm{~cm}^{-1}\right), \mathrm{C}-\mathrm{S}-\mathrm{C}$ str. $\left(741 \mathrm{~cm}^{-1}\right) ;{ }^{1} \mathrm{H}$ NMR $\left(\delta, \mathrm{CDCl}_{3}\right): 3.8\left(3 \mathrm{H}, \mathrm{s}, \mathrm{OCH}_{3}\right), 4.4(1 \mathrm{H}, \mathrm{s}, \mathrm{N}=\mathrm{CH}-), 6.8(1 \mathrm{H}, \mathrm{s}$, pyrimidine $\mathrm{CH}), 7.3-7.8$ (m, 5H, Ar-H), 8.5 (s, 1H, CO-NH); Anal. Calcd. For $\mathrm{C}_{23} \mathrm{H}_{16} \mathrm{O}_{2} \mathrm{~N}_{5} \mathrm{SCl}_{2} \mathrm{~F}: \mathrm{C}, 53.48 ; \mathrm{H}, 3.10 ; \mathrm{N}$, $13.56 \%$. Found: C, 53.44; H, 3.15; N, $13.50 \%$.

The remaining compounds were prepared by the above general method.

Preparation of 2-(4-methoxyphenyl)-3-[4-(2,4-dichloro-5-fluorophenyl)-6-(2-thienyl) pyrimidine-2-yl-ureido]-5H-4-thiazolidinone 5c. A solution of 1-(4-methoxy benzylidene)-4[4-(2,4-dichloro-5-fluorophenyl)-6-(2-thienyl)pyrimidine-2-yl] semicarbazide $4 \mathbf{c}(0.01 \mathrm{~mol})$ in dry benzene $(50.0 \mathrm{~mL})$ and mercaptoacetic acid $(0.012 \mathrm{~mol})$ was refluxed for $12 \mathrm{~h}$ using a DeanStark separator. After completion of reaction, excess of benzene was distilled off and the resulting product was treated with $5 \% \mathrm{NaHCO}_{3}$ solution to remove unreacted mercaptoacetic acid. The separated product was washed with water, dried and recrystallized from DMF:water as a creamish white solid in $62 \%$ yield. m.p. $139{ }^{\circ} \mathrm{C}$; IR $(\mathrm{KBr}): \mathrm{NH}\left(3223 \mathrm{~cm}^{-1}\right),=\mathrm{CH}$ str. $\left(3068 \mathrm{~cm}^{-}\right.$ $\left.{ }^{1}\right)$, 4-thiazolidinone moiety: $\mathrm{C}=\mathrm{O}\left(1674 \mathrm{~cm}^{-1}\right), \mathrm{C}=\mathrm{O}$ str. $\left(1642 \mathrm{~cm}^{-1}\right), \mathrm{C}-\mathrm{F}$ str. $\left(1056 \mathrm{~cm}^{-1}\right), \mathrm{C}-\mathrm{Cl}$ str. $\left(755 \mathrm{~cm}^{-1}\right), \mathrm{C}-\mathrm{S}-\mathrm{C}$ str. $\left(742 \mathrm{~cm}^{-1}\right) ;{ }^{1} \mathrm{H} \mathrm{NMR}\left(\delta, \mathrm{CDCl}_{3}\right): 3.6\left(1 \mathrm{H}, \mathrm{s}, \mathrm{SCH}_{2} \mathrm{CO}, 4-\right.$ thiazolidinone ring), $4.0\left(3 \mathrm{H}, \mathrm{s}, \mathrm{OCH}_{3}\right), 6.1(1 \mathrm{H}, \mathrm{s}, \mathrm{CH}$ of 4-thiazolidinone ring), $6.9(1 \mathrm{H}, \mathrm{s},-\mathrm{CH}$, pyrimidine ring), 7.3-7.8 (m, 9H, ArH), $8.6(1 \mathrm{H}, \mathrm{s}, \mathrm{CO}-\mathrm{NH})$; Anal. Calcd. for $\mathrm{C}_{25} \mathrm{H}_{18} \mathrm{O}_{3} \mathrm{~N}_{5} \mathrm{~S}_{2} \mathrm{Cl}{ }_{2} \mathrm{~F}$ : C, 50.8; H, 3.05; N, 11.86\%. Found: C, 50.5; H, 3.1; N, 11.83\%.

Similarly, compounds $\mathbf{5 a}, \mathbf{5 b}, \mathbf{5 d}, \mathbf{5 e}$ were prepared by the above general method.

2-(2-Chlorophenyl)-3-[4-(2,4-dichloro-5-fluorophenyl )-6-(2-thienyl)pyrimidine-2-yl-ureido] -5H-4-thiazolidinone 5 a.

Pale yellow solid in $67 \%$ yield. m.p. $145{ }^{\circ} \mathrm{C}$; IR (KBr): $-\mathrm{NH}\left(3220 \mathrm{~cm}^{-1}\right),=\mathrm{CH}$ str. $\left(3064 \mathrm{~cm}^{-1}\right)$, 4-thiazolidinone moiety: $\mathrm{C}=\mathrm{O}\left(1675 \mathrm{~cm}^{-1}\right), \mathrm{C}=\mathrm{O}$ str. $\left(1641 \mathrm{~cm}^{-1}\right), \mathrm{C}-\mathrm{F}$ str. $\left(1055 \mathrm{~cm}^{-1}\right), \mathrm{C}-\mathrm{Cl}$ str. $\left(754 \mathrm{~cm}^{-1}\right), \mathrm{C}-\mathrm{S}-\mathrm{C}$ str. $\left(741 \mathrm{~cm}^{-1}\right) ;{ }^{1} \mathrm{H} \mathrm{NMR}\left(\delta, \mathrm{CDCl}_{3}\right): 3.6\left(1 \mathrm{H}, \mathrm{s}, \mathrm{SCH}_{2} \mathrm{CO}, 4-\right.$ thiazolidinone ring), $6.0(1 \mathrm{H}, \mathrm{s}, \mathrm{CH}$ of 4-thiazolidinone ring), $6.8(1 \mathrm{H}, \mathrm{s}, \mathrm{CH}$, pyrimidine ring), 7.3-7.8 $(\mathrm{m}, 9 \mathrm{H}$, ArH), 8.5(1H, s, CO-NH); Anal. Calcd. for $\mathrm{C}_{24} \mathrm{H}_{15} \mathrm{O}_{2} \mathrm{~N}_{5} \mathrm{~S}_{2} \mathrm{Cl}_{3} \mathrm{~F}$ : C, 48.4; $\mathrm{H}, 2.52 ; \mathrm{N}, 11.77 \%$. Found: C, 48.2; H, 2.49; N, 11.73\%.

2-(2-Methoxyphenyl)-3-[4-(2,4-dichloro-5-fluorophenyl)-6-(2-thienyl)pyrimidine-2-ylureido]-5H-4-thiazolidinone $5 \mathrm{~b}$.

White solid in $70 \%$ yield. m.p. $128{ }^{\circ} \mathrm{C}$; IR $(\mathrm{KBr})$ : $\mathrm{NH}\left(3221 \mathrm{~cm}^{-1}\right),=\mathrm{CH}$ str. $\left(3068 \mathrm{~cm}^{-1}\right),{ }^{4-}$ thiazolidinone moiety: $\mathrm{C}=\mathrm{O}\left(1672 \mathrm{~cm}^{-1}\right), \mathrm{C}=\mathrm{O}$ str. $\left(1640 \mathrm{~cm}^{-1}\right), \mathrm{C}-\mathrm{F}$ str. $\left(1054 \mathrm{~cm}^{-1}\right), \mathrm{C}-\mathrm{Cl}$ str. $\left(754 \mathrm{~cm}^{-1}\right)$, C-S-C str. $\left(742 \mathrm{~cm}^{-1}\right) ;{ }^{1} \mathrm{H} \mathrm{NMR}\left(\delta, \mathrm{CDCl}_{3}\right): 3.6\left(1 \mathrm{H}, \mathrm{s}, \mathrm{SCH}_{2} \mathrm{CO}, 4\right.$-thiazolidinone ring), $4.1\left(3 \mathrm{H}, \mathrm{s}, \mathrm{OCH}_{3}\right), 6.2(1 \mathrm{H}, \mathrm{s}, \mathrm{CH}$ of 4-thiazolidinone ring $), 6.7(1 \mathrm{H}, \mathrm{s}, \mathrm{CH}$, pyrimidine ring), 7.3-7.9 (m, 9H, ArH), $8.4(1 \mathrm{H}, \mathrm{s}, \mathrm{CO}-\mathrm{NH})$; Anal. Calcd. for $\mathrm{C}_{25} \mathrm{H}_{18} \mathrm{O}_{3} \mathrm{~N}_{5} \mathrm{~S}_{2} \mathrm{Cl}_{2} \mathrm{~F}: \mathrm{C}, 50.8$; H, 3.05; N, 11.86\%. Found: C, 50.3; H, 3.0; N, 11.89\%.

2-(3,4,5-Trimethoxyphenyl)-3-[4-(2,4-dichloro-5-fluorophenyl)-6-(2-thienyl)pyrimidin-2-ylureido]-5H-4-thiazolidinone $5 \mathrm{~d}$.

Pale yellow solid in $76 \%$ yield. m.p. $103{ }^{\circ} \mathrm{C}$; IR $(\mathrm{KBr}):-\mathrm{NH}\left(3223 \mathrm{~cm}^{-1}\right),=\mathrm{CH}$ str. $\left(3066 \mathrm{~cm}^{-1}\right)$, 4-thiazolidinone moiety: $\mathrm{C}=\mathrm{O}\left(1673 \mathrm{~cm}^{-1}\right), \mathrm{C}=\mathrm{O}$ str. $\left(1644 \mathrm{~cm}^{-1}\right), \mathrm{C}-\mathrm{F}$ str. $\left(1054 \mathrm{~cm}^{-1}\right), \mathrm{C}-\mathrm{Cl}$ str. 
$\left(756 \mathrm{~cm}^{-1}\right)$, C-S-C str. $\left(741 \mathrm{~cm}^{-1}\right) ;{ }^{1} \mathrm{H}$ NMR $\left(\delta, \mathrm{CDCl}_{3}\right): 3.5\left(1 \mathrm{H}, \mathrm{s}, \mathrm{SCH}_{2} \mathrm{CO}, 4-\right.$ thiazolidinone ring), $4.0\left(9 \mathrm{H}, \mathrm{s}, 3,4,5\right.$-tri- $\left.\mathrm{OCH}_{3}\right), 6.1(1 \mathrm{H}, \mathrm{s}, \mathrm{CH}$ of 4-thiazolidinone ring $), 6.8(1 \mathrm{H}, \mathrm{s}, \mathrm{CH}$, pyrimidine ring), 7.3-7.8 (m, 7H, ArH), $8.4(1 \mathrm{H}, \mathrm{s}, \mathrm{CO}-\mathrm{NH})$; Anal. Calcd. for $\mathrm{C}_{27} \mathrm{H}_{22} \mathrm{O}_{5} \mathrm{~N}_{5} \mathrm{~S}_{2} \mathrm{Cl}_{2} \mathrm{~F}$ : C, 49.8; H, 3.38; N, 10.77\%. Found: C, 49.4; H, 3.41; N, 10.82\%.

2-(4-Fluorophenyl )-3-[4-(2,4-dichloro-5-fluorophenyl )-6-(2-thienyl) pyrimidin-2-yl-ureido] - 5H-4-thiazolidinone $5 \mathrm{e}$.

White solid in $73 \%$ yield. m.p. $130{ }^{\circ} \mathrm{C}$; IR $(\mathrm{KBr}):-\mathrm{NH}\left(3223 \mathrm{~cm}^{-1}\right),=\mathrm{CH}$ str. $\left(3068 \mathrm{~cm}^{-1}\right), 4-$ thiazolidinone moiety: $\mathrm{C}=\mathrm{O}\left(1674 \mathrm{~cm}^{-1}\right), \mathrm{C}=\mathrm{O}$ str. $\left(1642 \mathrm{~cm}^{-1}\right), \mathrm{C}-\mathrm{F}$ str. $\left(1056 \mathrm{~cm}^{-1}\right), \mathrm{C}-\mathrm{Cl}$ str. $\left(753 \mathrm{~cm}^{-1}\right)$, C-S-C str. $\left(742 \mathrm{~cm}^{-1}\right)$; ${ }^{1} \mathrm{H}$ NMR $\left(\delta, \mathrm{CDCl}_{3}\right): 3.7\left(1 \mathrm{H}, \mathrm{s}, \mathrm{SCH}_{2} \mathrm{CO}, 4-\right.$ thiazolidinone ring), $6.0(1 \mathrm{H}, \mathrm{s}, \mathrm{CH}$ of 4-thiazolidinone ring), $6.8(1 \mathrm{H}, \mathrm{s}, \mathrm{CH}$, pyrimidine ring), 7.3-7.8 $(\mathrm{m}, 9 \mathrm{H}$, ArH), 8.6 (1H, s, CO-NH); Anal. Calcd. for $\mathrm{C}_{24} \mathrm{H}_{15} \mathrm{O}_{2} \mathrm{~N}_{5} \mathrm{~S}_{2} \mathrm{Cl}_{2} \mathrm{~F}_{2}$ : C, 49.82; $\mathrm{H}, 2.59 ; \mathrm{N}, 12.11 \%$. Found: C, 49.78; H, 2.64; N, 12.08\%.

Preparation of 2-( 4-methoxyphenyl )-3-[ 4-( 2,4-dichloro-5-fluorophenyl )-6-( 2-thienyl ) pyrimidine-2-yl-ureido]-5-methyl-4-thiazolidinone 6c. A solution of 1-(4-methoxy benzylidene)-4 - [4-(2,4-dichloro-5-fluorophenyl)-6-(2-thienyl)pyrimidine-2-yl] semicarbazide 4 c $(0.01 \mathrm{~mol})$ in dry benzene $(50.0 \mathrm{~mL})$ and 2-mercaptopropanoic acid $(0.012 \mathrm{~mol})$ refluxed for $14 \mathrm{~h}$ using a Dean-Stark separator. After completion of the reaction, excess of benzene was removed by distillation and the product was treated with $5 \% \mathrm{NaHCO}_{3}$ solution to remove unreacted 2-mercaptopropanoic acid. The separated product was washed with water, dried and recrystallized from chloroform: methanol (1:1) mixture as a white solid in 72\% yield. m.p. 101 ${ }^{\circ} \mathrm{C}$; IR (KBr): $\mathrm{NH}\left(3223 \mathrm{~cm}^{-1}\right), 4-$ thiazolidinone moiety; $\mathrm{C}=\mathrm{O}\left(1674 \mathrm{~cm}^{-1}\right), \mathrm{C}=\mathrm{O}$ str. $\left(1642 \mathrm{~cm}^{-1}\right)$, C-F str. $\left(1056 \mathrm{~cm}^{-1}\right), \mathrm{C}-\mathrm{S}-\mathrm{C}$ str. $\left(754 \mathrm{~cm}^{-1}\right) ;{ }^{1} \mathrm{H} \mathrm{NMR}\left(\delta, \mathrm{CDCl}_{3}\right): 1.5\left(3 \mathrm{H}, \mathrm{d}, \mathrm{CHCH}_{3}\right), 3.9(3 \mathrm{H}, \mathrm{s}$, $\left.\mathrm{CH}_{3}\right), 4.20\left(1 \mathrm{H}, \mathrm{q}, \mathrm{C} \underline{\mathrm{H}}-\mathrm{CH}_{3}\right), 5.96(1 \mathrm{H}, \mathrm{s}, \mathrm{CH}$ of 4-thiazolidinone ring), $6.8(1 \mathrm{H}, \mathrm{s}, \mathrm{CH}$, pyrimidine ring), 7.3-7.8 (m, $9 \mathrm{H}, \mathrm{ArH}), 8.7(1 \mathrm{H}, \mathrm{s}, \mathrm{CO}-\mathrm{NH})$; Anal. Calcd. for $\mathrm{C}_{26} \mathrm{H}_{20} \mathrm{O}_{3} \mathrm{~N}_{5} \mathrm{~S}_{2} \mathrm{Cl}_{2} \mathrm{~F}$ : C, 51.65; H, 3.31; N, 11.58\%. Found: C, 51.61; H, 3.29; N, 11.62\%.

Similarly, compounds $\mathbf{6 a}, \mathbf{6 b}, \mathbf{6 d}, \mathbf{6 e}$ were prepared by the above general method.

\section{2-(2-Chlorophenyl)-3-[4-(2,4-dichloro-5-fluorophenyl)-6-(2-thienyl)pyrimidine-2-yl-urei-}

\section{do]-5-methyl-4-thiazolidinone 6 a.}

Colorless solid in $68 \%$ yield. m.p. $121^{\circ} \mathrm{C}$; IR ( $\left.\mathrm{KBr}\right)$ :-NH $\left(3220 \mathrm{~cm}^{-1}\right)$, 4-thiazolidinone moiety; $\mathrm{C}=\mathrm{O}\left(1674 \mathrm{c} \mathrm{m}^{-1}\right), \mathrm{C}=\mathrm{O}$ str. $\left(1641 \mathrm{~cm}^{-1}\right), \mathrm{C}-\mathrm{F}$ str. $\left(1052 \mathrm{~cm}^{-1}\right), \mathrm{C}-\mathrm{S}-\mathrm{C}$ str. $\left(752 \mathrm{~cm}^{-1}\right) ;{ }^{1} \mathrm{H} \mathrm{NMR}(\delta$, $\left.\mathrm{CDCl}_{3}\right): 1.6\left(3 \mathrm{H}, \mathrm{d}, \mathrm{CHCH}_{3}\right), 3.6\left(1 \mathrm{H}, \mathrm{s}, \mathrm{SCH}_{2} \mathrm{CO}, 4\right.$-thiazolidinone ring), $4.2\left(1 \mathrm{H}, \mathrm{q}, \mathrm{C} \underline{\mathrm{H}}-\mathrm{CH}_{3}\right)$, $5.9(1 \mathrm{H}, \mathrm{s}, \mathrm{CH}$ of 4-thiazolidinone ring), $6.7(1 \mathrm{H}, \mathrm{s}, \mathrm{CH}$, pyrimidine ring), 7.3-7.8 $(\mathrm{m}, 9 \mathrm{H}, \mathrm{r}-\mathrm{H})$, $8.6(1 \mathrm{H}, \mathrm{s}, \mathrm{CO}-\mathrm{NH})$. Anal. Calcd. for $\mathrm{C}_{25} \mathrm{H}_{17} \mathrm{O}_{2} \mathrm{~N}_{5} \mathrm{~S}_{2} \mathrm{Cl}_{3} \mathrm{~F}$ : C, 49.3; H, 2.79; N, 11.5\%. Found: C, 49.7; H, 2.75; N, 11.53\%.

2-( 2-Methoxyphenyl )-3 -[ 4-( 2,4-dichloro-5-fluorophenyl )-6-(2-thienyl ) pyrimidine-2-ylureido]-5-methyl-4-thiazolidinone $6 \mathrm{~b}$.

Off white solid in $61 \%$ yield. m.p. $152{ }^{\circ} \mathrm{C}$; IR (KBr): $\mathrm{NH}\left(3223 \mathrm{~cm}^{-1}\right)$, 4-thiazolidinone moiety; $\mathrm{C}=\mathrm{O}\left(1672 \mathrm{~cm}^{-1}\right), \mathrm{C}=\mathrm{O}$ str. $\left(1640 \mathrm{~cm}^{-1}\right), \mathrm{C}-\mathrm{F}$ str. $\left(1056 \mathrm{~cm}^{-1}\right), \mathrm{C}-\mathrm{S}-\mathrm{C}$ str. $\left(754 \mathrm{~cm}^{-1}\right) ;{ }^{1} \mathrm{H}$ NMR $(\delta$, $\left.\mathrm{CDCl}_{3}\right): 1.7\left(3 \mathrm{H}, \mathrm{d}, \mathrm{CHCH}_{3}\right), 4.0\left(3 \mathrm{H}, \mathrm{s}, \mathrm{OCH}_{3}\right), 4.3\left(1 \mathrm{H}, \mathrm{q}, \mathrm{C} \underline{\mathrm{H}}-\mathrm{CH}_{3}\right), 5.8(1 \mathrm{H}, \mathrm{s}, \mathrm{CH}$ of $4-$ thiazolidinone ring), $6.8(1 \mathrm{H}, \mathrm{s}, \mathrm{CH}$, pyrimidine ring $), 7.3-7.7(\mathrm{~m}, 9 \mathrm{H}, \mathrm{ArH}), 8.5(1 \mathrm{H}, \mathrm{s}, \mathrm{CO}-$ 
$\mathrm{NH}$ ); Anal. Calcd.for $\mathrm{C}_{26} \mathrm{H}_{20} \mathrm{O}_{3} \mathrm{~N}_{5} \mathrm{~S}_{2} \mathrm{Cl}_{2} \mathrm{~F}$ : C, 51.65; H, 3.31; N, 11.58\%. Found: C, 51.68; H, $3.28 ; \mathrm{N}, 11.55 \%$.

2-(3,4,5-Trimethoxyphenyl)-3-[4-(2,4-dichloro-5-fluorophenyl)-6-(2-thienyl)pyrimidine-2yl-ureido]-5-methyl-4-thiazolidinone $6 \mathrm{~d}$.

Light yellow solid in $79 \%$ yield. m.p. $123{ }^{\circ} \mathrm{C}$; IR (KBr):-NH $\left(3222 \mathrm{~cm}^{-1}\right)$, 4-thiazolidinone moiety: $\mathrm{C}=\mathrm{O}\left(1672 \mathrm{~cm}^{-1}\right), \mathrm{C}=\mathrm{O}$ str. $\left(1641 \mathrm{~cm}^{-1}\right), \mathrm{C}-\mathrm{F}$ str. $\left(1053 \mathrm{~cm}^{-1}\right), \mathrm{C}-\mathrm{S}-\mathrm{C}$ str. $\left(753 \mathrm{~cm}^{-1}\right) ;{ }^{1} \mathrm{H}$ NMR $\left(\delta, \mathrm{CDCl}_{3}\right): 1.6\left(3 \mathrm{H}, \mathrm{d}, \mathrm{CHCH}_{3}\right), 3.9\left(9 \mathrm{H}, \mathrm{s}, 3,4,5-\right.$ tri- $\left.\mathrm{OCH}_{3}\right), 4.2\left(1 \mathrm{H}, \mathrm{q}, \mathrm{C}-\mathrm{H}_{-} \mathrm{CH}_{3}\right), 5.8$ $(1 \mathrm{H}, \mathrm{s}, \mathrm{CH}$ of 4-thiazolidinone ring), $6.7(1 \mathrm{H}, \mathrm{s}, \mathrm{CH}$, pyrimidine ring), 7.3-7.8 $(\mathrm{m}, 7 \mathrm{H}, \mathrm{ArH}), 8.7$ $(1 \mathrm{H}, \mathrm{s}, \mathrm{CO}-\mathrm{NH})$; Anal. Calcd. for $\mathrm{C}_{28} \mathrm{H}_{24} \mathrm{O}_{5} \mathrm{~N}_{5} \mathrm{~S}_{2} \mathrm{Cl}_{2} \mathrm{~F}$ : C, 50.6; H, 3.61; N, 10.54\%. Found: C, $50.57 ; \mathrm{H}, 3.58 ; \mathrm{N}, 10.59 \%$.

2-(4-Fluorophenyl)-3-[4-(2,4-dichloro-5-fluorophenyl)-6-(2-thienyl)pyrimidine-2-yl-ureido]5-methyl-4-thiazolidinone 6e.

White solid in $68 \%$ yield. m.p. $141{ }^{\circ} \mathrm{C}$; IR $(\mathrm{KBr}):-\mathrm{NH}\left(3224 \mathrm{~cm}^{-1}\right)$, 4-thiazolidinone moiety: $\mathrm{C}=\mathrm{O}\left(1673 \mathrm{~cm}^{-1}\right), \mathrm{C}=\mathrm{O}$ str. $\left(1640 \mathrm{~cm}^{-1}\right), \mathrm{C}-\mathrm{F}$ str. $\left(1055 \mathrm{~cm}^{-1}\right), \mathrm{C}-\mathrm{S}-\mathrm{C}$ str. $\left(752 \mathrm{~cm}^{-1}\right) ;{ }^{1} \mathrm{H}$ NMR $(\delta$, $\left.\mathrm{CDCl}_{3}\right): 1.6\left(3 \mathrm{H}, \mathrm{d}, \mathrm{CHCH}_{3}\right), 4.2\left(1 \mathrm{H}, \mathrm{q}, \mathrm{C} \underline{\mathrm{H}}-\mathrm{CH}_{3}\right), 5.7(1 \mathrm{H}, \mathrm{s}, \mathrm{CH}$ of 4-thiazolidinone ring $), 6.9$ $(1 \mathrm{H}, \mathrm{s}, \mathrm{CH}$, pyrimidine ring), 7.4-7.8 (m, 9H, $\mathrm{ArH}), 8.6(1 \mathrm{H}, \mathrm{s}, \mathrm{CO}-\mathrm{NH})$; Anal. Calcd. for $\mathrm{C}_{25} \mathrm{H}_{17} \mathrm{O}_{2} \mathrm{~N}_{5} \mathrm{~S}_{2} \mathrm{Cl}_{2} \mathrm{~F}_{2}$ : C, 50.67; H, 2.87; N, 11.82\%. Found: C, 50.71; H, 2.84; N, 11.87\%.

2-(4-Methoxyphenyl)-3-[4-(2,4-dichloro-5-fluorophenyl)-6-(2-thienyl)pyrimidine-2-yl-ureido]-5-carboxymethyl-4-thiazolidinone 7c. A solution of 1-(4-methoxy benzylidene)-4-[4-(2,4dichloro-5-fluorophenyl)-6-(2-thienyl)pyrimidine-2-yl] semicarbazide 4c $(0.01 \mathrm{~mol})$ in dry benzene $(50.0 \mathrm{~mL})$ and mercaptosuccinic acid $(0.012 \mathrm{~mol})$ was refluxed for $13 \mathrm{~h}$ using DeanStark separator. After completion of reaction, excess benzene was removed by distillation. The resulting product was treated with $5 \% \mathrm{NaHCO}_{3}$ solution to remove unreacted mercaptosuccinic acid. The separated product was washed with water, dried and recrystallized from chloroform/acetone as a pale yellow solid in $76 \%$ yield. m.p. $136{ }^{\circ} \mathrm{C}$; IR $(\mathrm{KBr}): \mathrm{NH}\left(3223 \mathrm{~cm}^{-1}\right)$, 4-thiazolidinone; $\mathrm{C}=\mathrm{O}\left(1674 \mathrm{~cm}^{-1}\right), \mathrm{C}=\mathrm{O}$ str. $\left(1642 \mathrm{~cm}^{-1}\right), \mathrm{C}-\mathrm{F}$ str. $\left(1056 \mathrm{~cm}^{-1}\right), \mathrm{C}-\mathrm{Cl}$ str. $(758$ $\left.\mathrm{cm}^{-1}\right)$, C-S-C str. $\left(740 \mathrm{~cm}^{-1}\right) ;{ }^{1} \mathrm{H}$ NMR $\left(\delta, \mathrm{CDCl}_{3}\right): 2.6\left(1 \mathrm{H}, \mathrm{d}, \mathrm{CH}_{\mathrm{A}} \mathrm{COOH}\right), 3.0(1 \mathrm{H}, \mathrm{d}$, $\left.\mathrm{CH}_{\mathrm{B}} \mathrm{COOH}\right), 3.9\left(3 \mathrm{H}, \mathrm{s}, \mathrm{OCH}_{3}\right), 6.2(1 \mathrm{H}, \mathrm{s}, \mathrm{CH}$ of 4-thiazolidinone ring $), 6.9(1 \mathrm{H}, \mathrm{s}, \mathrm{CH}$, pyrimidine ring), 7.3-7.9 (m, 9H, ArH), $8.6(1 \mathrm{H}, \mathrm{s}, \mathrm{CO}-\mathrm{NH})$; Anal. Calcd. for $\mathrm{C}_{27} \mathrm{H}_{20} \mathrm{O}_{5} \mathrm{~N}_{5} \mathrm{~S}_{2} \mathrm{Cl}_{2} \mathrm{~F}$ : C, 50.0; H, 3.08; N, 10.8\%. Found: C, 50.03; H, 3.04; N, $10.83 \%$.

The remaining compounds $\mathbf{7 a}, \mathbf{7 b}, \mathbf{7 d}, \mathbf{7 e}$ were synthesised by the same procedure.

2-(2-Chlorophenyl)-3-[4-(2,4-dichloro-5-fluorophenyl)-6-(2-thienyl)pyrimidine-2-yl-ureido] -5-carboxymethyl-4-thiazolidinone 7a.

White solid in $67 \%$ yield. m.p. $129^{\circ} \mathrm{C}$; IR $(\mathrm{KBr})$ : $-\mathrm{NH}\left(3219 \mathrm{~cm}^{-1}\right)$, 4-thiazolidinone: $\mathrm{C}=\mathrm{O}(1674$ $\left.\mathrm{cm}^{-1}\right), \mathrm{C}=\mathrm{O}$ str. $\left(1640 \mathrm{~cm}^{-1}\right), \mathrm{C}-\mathrm{F}$ str. $\left(1052 \mathrm{~cm}^{-1}\right), \mathrm{C}-\mathrm{Cl}$ str. $\left(756 \mathrm{~cm}^{-1}\right), \mathrm{C}-\mathrm{S}-\mathrm{C}$ str. $\left(741 \mathrm{~cm}^{-1}\right) ;{ }^{1} \mathrm{H}$ $\operatorname{NMR}\left(\delta, \mathrm{CDCl}_{3}\right): 2.65\left(1 \mathrm{H}, \mathrm{d}, \mathrm{CH}_{\mathrm{A}} \mathrm{COOH}\right), 2.85\left(1 \mathrm{H}, \mathrm{d}, \mathrm{CH}_{\mathrm{B}} \mathrm{COOH}\right), 6.1(1 \mathrm{H}, \mathrm{s}, \mathrm{CH}$ of $4-$ thiazolidinone ring), $6.8(1 \mathrm{H}, \mathrm{s}, \mathrm{CH}$, pyrimidine ring), 7.3-7.8 (m, 9H, $\mathrm{ArH}), 8.7(1 \mathrm{H}, \mathrm{s}, \mathrm{CO}-$ $\mathrm{NH}$ ); Anal. Calcd. for $\mathrm{C}_{26} \mathrm{H}_{17} \mathrm{O}_{4} \mathrm{~N}_{5} \mathrm{~S}_{2} \mathrm{Cl}{ }_{3} \mathrm{~F}$ : C, 47.8; H, 2.60; N, 10.72\%. Found: C, 47.77; H, $2.55 ; \mathrm{N}, 10.77 \%$. 
2-(2-Methoxyphenyl)-3-[4-(2,4-dichloro-5-fluorophenyl)-6-(2-thienyl)pyrimidine-2-yl-ureido]-5-carboxymethyl-4-thiazolidinone $7 \mathrm{~b}$.

Creamish white solid in $64 \%$ yield. m.p. $132{ }^{\circ} \mathrm{C}$; IR $(\mathrm{KBr})$ : $-\mathrm{NH}\left(3222 \mathrm{~cm}^{-1}\right)$, 4thiazolidinone $\mathrm{C}=\mathrm{O}\left(1672 \mathrm{~cm}^{-1}\right), \mathrm{C}=\mathrm{O}$ str. $\left(1641 \mathrm{~cm}^{-1}\right), \mathrm{C}-\mathrm{F}$ str. $\left(1052 \mathrm{~cm}^{-1}\right), \mathrm{C}-\mathrm{Cl}$ str. $\left(753 \mathrm{~cm}^{-1}\right)$, C-S-C str. $\left(741 \mathrm{~cm}^{-1}\right)$; ${ }^{1} \mathrm{H} \mathrm{NMR}\left(\delta, \mathrm{CDCl}_{3}\right): 2.6\left(1 \mathrm{H}, \mathrm{d}, \mathrm{CH}_{\mathrm{A}} \mathrm{COOH}\right), 2.9\left(1 \mathrm{H}, \mathrm{d}, \mathrm{CH}_{\mathrm{B}} \mathrm{COOH}\right)$, $4.0\left(3 \mathrm{H}, \mathrm{s}, \mathrm{OCH}_{3}\right), 6.2(1 \mathrm{H}, \mathrm{s}, \mathrm{CH}$ of 4-thiazolidinone ring), $6.8(1 \mathrm{H}, \mathrm{s}, \mathrm{CH}$, pyrimidine ring), 7.3-7.8 (m, 9H, ArH), $8.5(1 \mathrm{H}, \mathrm{s}, \mathrm{CO}-\mathrm{NH})$; Anal. Calcd. for $\mathrm{C}_{27} \mathrm{H}_{20} \mathrm{O}_{5} \mathrm{~N}_{5} \mathrm{~S}_{2} \mathrm{Cl}_{2} \mathrm{~F}$ : C, 50.0; $\mathrm{H}$, 3.08 ; N, 10.8\%. Found: C, 50.05; H, 3.1; N, 10.85\%.

2-(3,4,5-Trimethoxyphenyl-3-[4-(2,4-dichloro-5-fluorophenyl)-6-(2-thienyl)pyrimidine-2-ylureido]-5-carboxymethyl-4-thiazolidinone $7 \mathrm{~d}$.

Almost white solid in $66 \%$ yield. m.p. $119^{\circ} \mathrm{C}$; IR (KBr): $-\mathrm{NH}\left(3221 \mathrm{~cm}^{-1}\right)$, 4-thiazolidinone: $\mathrm{C}=\mathrm{O}\left(1672 \mathrm{~cm}^{-1}\right), \mathrm{C}=\mathrm{O}$ str.(1643 $\left.\mathrm{cm}^{-1}\right), \mathrm{C}-\mathrm{F}$ str.(1053 $\left.\mathrm{cm}^{-1}\right), \mathrm{C}-\mathrm{Cl}$ str. $\left(753 \mathrm{~cm}^{-1}\right), \mathrm{C}-\mathrm{S}-\mathrm{C}$ str. $(742$ $\left.\mathrm{cm}^{-1}\right) ;{ }^{1} \mathrm{H}$ NMR $\left(\delta, \mathrm{CDCl}_{3}\right): 2.6\left(1 \mathrm{H}, \mathrm{d}, \mathrm{CH}_{\mathrm{A}} \mathrm{COOH}\right), 3.0\left(1 \mathrm{H}, \mathrm{d}, \mathrm{CH}_{\mathrm{B}} \mathrm{COOH}\right), 4.1(9 \mathrm{H}, \mathrm{s}, 3,4,5-$ tri- $\left.\mathrm{OCH}_{3}\right), 6.2(1 \mathrm{H}, \mathrm{s}, \mathrm{CH}$ of 4-thiazolidinone ring), $6.8(1 \mathrm{H}, \mathrm{s}, \mathrm{CH}$, pyrimidine ring), 7.4-7.8 (m, $7 \mathrm{H}, \mathrm{ArH}), 8.7$ (1H, s, CO-NH); Anal. Calcd. for $\mathrm{C}_{29} \mathrm{H}_{24} \mathrm{O}_{7} \mathrm{~N}_{5} \mathrm{~S}_{2} \mathrm{Cl}_{2} \mathrm{~F}: \mathrm{C}, 49.15 ; \mathrm{H}, 3.38 ; \mathrm{N}$, 9.88\%. Found: C, 49.11; H, 3.34; N, 9.91\%.

2-(4-Fluorophenyl)-3-[4-(2,4-dichloro-5-fluorophenyl)-6-(2-thienyl)pyrimidine-2-yl-ureido]5-carboxymethyl-4-thiazolidinone $7 \mathrm{e}$.

Off-white solid in $81 \%$ yield. m.p. $142{ }^{\circ} \mathrm{C}$; IR $(\mathrm{KBr})$ : $\mathrm{NH}\left(3223 \mathrm{~cm}^{-1}\right)$, 4-thiazolidinone; $\mathrm{C}=\mathrm{O}$ $\left(1674 \mathrm{~cm}^{-1}\right), \mathrm{C}=\mathrm{O}$ str. $\left(1640 \mathrm{~cm}^{-1}\right), \mathrm{C}-\mathrm{F}$ str. $\left(1055 \mathrm{~cm}^{-1}\right), \mathrm{C}-\mathrm{Cl} \mathrm{str} .\left(756 \mathrm{~cm}^{-1}\right), \mathrm{C}-\mathrm{S}-\mathrm{C}$ str. $\left(743 \mathrm{~cm}^{-}\right.$ $\left.{ }^{1}\right) ;{ }^{1} \mathrm{H}$ NMR $\left(\delta, \mathrm{CDCl}_{3}\right): 2.6\left(1 \mathrm{H}, \mathrm{d}, \mathrm{CH}_{\mathrm{A}} \mathrm{COOH}\right), 2.9\left(1 \mathrm{H}, \mathrm{d}, \mathrm{CH}_{\mathrm{B}} \mathrm{COOH}\right), 6.1(1 \mathrm{H}, \mathrm{s}, \mathrm{CH}$ of 4thiazolidinone ring), $6.7(1 \mathrm{H}, \mathrm{s}, \mathrm{CH}$, pyrimidine ring), 7.4-7.9 (m, 9H, $\mathrm{ArH}), 8.7(1 \mathrm{H}, \mathrm{s}, \mathrm{CO}-$ $\mathrm{NH}$ ); Anal. Calcd. for $\mathrm{C}_{26} \mathrm{H}_{17} \mathrm{O}_{4} \mathrm{~N}_{5} \mathrm{~S}_{2} \mathrm{Cl}_{2} \mathrm{~F}_{2}$ : C, 49.05; H, 2.67; N, 11.0\%. Found: C, 49.11; H, $2.71 ; \mathrm{N}, 11.04 \%$.

\section{Acknowledgements}

The authors thank Department of Chemistry, B.K.M. Science College, Valsad for research facilities, Astra Zeneca Pharma India Limited, Bangalore for ${ }^{1} \mathrm{H}$ NMR spectral analysis, and Choksi Laboratories Limited, Vapi for providing IR Spectral analysis.

\section{References}

1. Berreca, M. L.; Balzarini, J.; Chimirri, A.; Clercq, E. De.; Luca, L. D.; Holtije, A. M.; Monforte, P.; Pannecouque, C.; Rao, A.; Zappala, M. M. J. Med. Chem, 2002, 45, 5410.

2. Grasso, S.; Chimirri, A.; Monforte, P.; Fenech, G.; Zappalar, M.; Monforte, A. M. Farmaco Ed. Sci. 1988, 43, 851, Chem. Abstr, 1989, 110, 50734c.

3. Shrivastava, V. K.; Singh, S.; Gulatia, A.; Shankar, K. Indian J. Chem. 1987, $26 B, 652$. 
4. Viltaria, D. M.; Orazio, M.; Eugenio, P.; Anlonia, C.; Federico, G.; Adde, B. J. Med. Chem. 1992, 35, 2910.

5. Pandya, D.; Nair, K.B.; Pharmazie, 1993, 48, 414, Chem.Abstr, 1994, 120,106843p.

6. Mahasafi, M. A.; Meshkatalsadat, M. H.; Parekh, H. Indian J. Chem. 1987, 26B, 803.

7. Claris, D. A. S. African Z. 1991, 92, 849, Chem. Abstr. 1993, 119, 34322x.

8. Bjune, K.; Stubhaug, A.; Dodgson, M. S.; Breirik, H. Acta Anaestheriol. Scand. 1996, 40, 399, Chem. Abstr. 1996, 125, 491.

9. Nargund, L. V. G.; Reddy G. R. N.; Hariprasad, V. Indian J. Chem. 1996, 35B, 499.

10. Fahmy, A. M.; Hassan, K. M. A.; Khalaf, A.; Ahmed, R. A. Indian J. Chem. 1987, $26 B, 884$.

11. Dawey, W.; Tivey, D. J., J. Chem. Soc. 1958, 1320.

12. Kohler, H. M.; Chadwell, H.; Gilman, H.; Blatt, A. H., (Eds) Org. Synth.; Wiley: New York, 1967, Coll. Vol.I, p 78.

13. Mehra, H. S. J. Indian Chem. Soc. 1968, 45,178.

14. Barry, A. L.; The Antimicrobial Susceptibility Test: Principle and Practice, ( Illuslea \& Febiger, Philadelphia), 1976, p. 180 [Biol.Abstr. 1977, 64, 25183].

15. National committee for clinical laboratory standards, 1997, Reference method for broth dilution antifungal susceptibility testing of yeast. Approved standard NCCLS document; 720 M27 A. (ISBN 156238328 0, ISSN 0273 3099). National committee for clinical laboratory standards, 940, West Valley Road, Suite 1400, Wayne, Pennsylvania, 19807.

16. Vogel, A. I., Elementary Practical Organic Chemistry, Part III, Longman London, 1975, 625 . 